\title{
A Locally Finite Model for Gravity
}

\author{
Gerard 't Hooft
}

Received: 26 June 2008 / Accepted: 8 July 2008 / Published online: 24 July 2008

(C) The Author(s) 2008. This article is published with open access at Springerlink.com

\begin{abstract}
Matter interacting classically with gravity in $3+1$ dimensions usually gives rise to a continuum of degrees of freedom, so that, in any attempt to quantize the theory, ultraviolet divergences are nearly inevitable. Here, we investigate matter of a form that only displays a finite number of degrees of freedom in compact sections of space-time. In finite domains, one has only exact, analytic solutions. This is achieved by limiting ourselves to straight pieces of string, surrounded by locally flat sections of space-time. Globally, however, the model is not finite, because solutions tend to generate infinite fractals. The model is not (yet) quantized, but could serve as an interesting setting for analytical approaches to classical general relativity, as well as a possible stepping stone for quantum models. Details of its properties are explained, but some problems remain unsolved, such as a complete description of the most violent interactions, which can become quite complex.
\end{abstract}

Keywords Locally flat $\cdot$ Straight strings $\cdot$ Holonomy $\cdot$ Classical general relativity Exact solutions

\section{Introduction: Gravity in $2+1$ Dimensions}

Classical point particles, interacting only gravitationally in $2+1$ dimensions, require a limited number of physical degrees of freedom per particle [1-3]. Although they are surrounded by locally flat space-time (if the cosmological constant is taken to be zero), space-time may globally form a closed, compact universe [4, 5]. The classical equations can be solved exactly, and for this reason this is a magnificent model for a

G. 't Hooft (凶)

Spinoza Institute, P.O. Box 80.195, 3508 Utrecht, The Netherlands

e-mail: g.thooft@uu.nl

url: http://www.phys.uu.nl/ thooft 
Fig. 1 (a) 2 dimensional space surrounding a point particle. Points $A$ and $A^{\prime}$ are identified. (b) artist's impression of 2 dimensional space embedded in higher dimensions

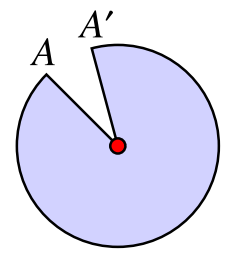

a)

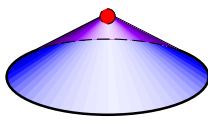

b)

complete, exactly solvable cosmology. Many researchers are more interested in quantized theories, where the point particles are removed as being unwanted topological defects, to be replaced by non-trivial global topological features of the universe. Such theories however have no local degrees of freedom at all, so from a conceptual point of view they are actually further removed from the physical world than our gravitating point particles.

Indeed, the importance of the model of gravitating point particles in a locally flat $2+1$ dimensional space-time, is still severely underestimated. It will serve as a starting point for the $3+1$ dimensional theory discussed in this paper. In $2+1$ dimensions, pure gravity (gravity without matter in some small section of space-time) has no physical degrees of freedom at all. This is because the Riemann curvature $R_{\alpha \beta \gamma \delta}$ can be rewritten in terms of a symmetric $3 \times 3$ matrix $Q^{\mu \nu}$ as follows:

$$
R_{\alpha \beta \gamma \delta}=\varepsilon_{\alpha \beta \mu} \varepsilon_{\gamma \delta \nu} Q^{\mu \nu}
$$

so that the Ricci curvature is

$$
\begin{aligned}
& R_{\alpha \gamma}=R_{\alpha \beta \gamma}{ }^{\beta}=\left(g_{\mu \nu} g_{\alpha \gamma}-g_{\mu \gamma} g_{\alpha \nu}\right) Q^{\mu \nu}=Q_{\mu}^{\mu} g_{\alpha \gamma}-Q_{\gamma \alpha} \\
& \quad \text { so that } Q_{\mu \nu}=\frac{1}{2} R_{\alpha}^{\alpha} g_{\mu \nu}-R_{\mu \nu} .
\end{aligned}
$$

Clearly, if matter is absent, $R_{\mu \nu}$ vanishes, and therefore so do $Q_{\mu \nu}$ and $R_{\alpha \beta \gamma \delta}$. Conversely, a point particle represents point curvature. Thus, particles are point singularities surrounded by flat space-time. A particle at rest can be described as in Fig. 1. The wedge is stitched closed, so that the points $A$ and $A^{\prime}$ are identified. The defect angle can directly be identified with the particle's rest mass. One can show that systems containing several point particles in motion, while the total momentum is kept zero, is surrounded by a conical space-time, of which the deficit angle can be identified with the total energy. ${ }^{1}$ Only particles with negative rest mass, or systems with negative energy, are surrounded by a conical space-time with negative deficit angle, or surplus angle, see also Fig. 2 b.

When such a particle is set in motion, the surrounding space-time is described by performing Lorentz transformations upon the stationary case of Fig. 1. One then can

\footnotetext{
${ }^{1}$ There may however also be a time shift when a curve is followed around the cone. This time shift is then identified with total angular momentum.
} 
study systems with many particles, by viewing space-time as a tessellation of locally flat triangles, or polygons. A number of surprises are encountered:

- Using fast moving, heavy particles, a space-time can be created that appears to allow for the existence of closed timelike curves [6, 7], much like in Gödel's universe [8]. This would clash with fundamental principles of causality, but one can also show that in physically realistic models such configurations cannot occur, because a universe that contains such a "Gott pair" would actually collapse to a point before the timelike curve could be closed [9]. Any closed timelike curve that one would be tempted to construct would pass through a non-existing region of the universe.

- If all particles in a $2+1$ dimensional universe would be stationary or nearly stationary, the two-dimensional integrated scalar Ricci tensor, $\int \delta^{2} x \sqrt{g} R$, would have to be positive, so with a sufficient number of particles the spacelike part of this universe would always close into an $S(2)$ geometry. Its timelike coordinate can form a compact dimension (featuring both a bang and a crunch), or a semi-infinite one, with either a bang or a crunch.

- With fast moving particles one can however also form 2-d surfaces with higher genus, without requiring negative mass particles [10].

Quantization of this system is often carried out 'as usual' [11, 12], but there are delicate problems, having to do with the fact that we are dealing with a strictly finite universe, so that the role of an 'observer' is questionable, and the statistical interpretation of the wave function is dubious because the finiteness of the universe prohibits infinite sequences of experiments to which a statistical analysis would apply. Carrying quantization out with care, one first observes that evidently, time is quantized into 'Planck time' units [13]. This is easily derived from the fact that the Hamiltonian is an angle and it is bounded to the unit circle. Consequently, a quantum theory cannot be formulated using differential equations in time, but rather one should use evolution operators that bridge integral time segments. This indeed can be regarded as a first indication of some sort of space-time discreteness, which we will encounter later in a more concrete way. However, a confrontation with foundational aspects of quantum mechanics appears to be inevitable.

In this paper, the question is asked whether a similar "finite" theory can also be formulated in $3+1$ dimensions. This is far from obvious. One first notices that the absence of matter now no longer guarantees local flatness, since the Ricci curvature $R_{\mu \nu}$ can vanish without the total Riemann curvature $R_{\beta \mu \nu}^{\alpha}$ being zero. However, one still can decide to view space-time as a tessellation of locally flat pieces. The defects in such a construction again may represent matter. The primary defects one finds are direct generalizations of the $2+1$ dimensional case. Take a particle-like defect in 2 -space. In $3+1$ dimensions, such defects manifest themselves as strings.

Our starting point is that, indeed, straight strings are surrounded by a locally flat metric. In Sect. 2, we recapitulate the well-known derivation of the metric near such a string. Then, in Sect. 3, moving strings are described. These topics are quite elementary but we need these discussions to initiate the mathematical derivations that come next. In Sect. 4, joints between strings are introduced. Then we arrive at collisions. The orthogonal case (Sect. 5) seems to be the easiest case, though we will see that 
there is a catch, at the end. The most difficult case occurs when two strings approach at an angle. There are two possibilities. The "quadrangle" final state is discussed in Sect. 6. It leads to delicate mathematical relations between $S L(2, C)$ matrices. We needed to do some computer algebra, the result of which was deferred to the Appendix. This algebra reveals that, at the highest relative velocities of the approaching strings, the quadrangle final state is ruled out, so that more complex final states are expected. An attempt to describe these is made in Sect. 7.

\section{Strings}

It could be that matter is always arranged in such a way that it can be regarded as defects in a locally perfectly flat space-time. As opposed to technical approaches towards solving General Relativity [14], we now regard matter of this form as elementary. The novelty in this idea is that, in spite of matter being distributed on subspaces of measure zero, we still insist that it obeys local laws of causal behavior. Let us see how this looks.

By simply adding the third space dimension as a spectator, orthogonal to the first two, we find flat 3 dimensional space-time surrounding a string. Indeed, the energy momentum tensor of a straight, infinite, static string pointing in the $z$-direction, is

$$
T_{\mu \nu}=t_{\mu \nu} \delta^{2}(\tilde{x}), \quad t_{33}=-t_{00}=\varrho, \text { all other } t_{\alpha \beta}=0
$$

where $\varrho$ is the string tension parameter. When $\varrho$ is small, the metric generated by such a string is found by slightly smearing the delta peak. The curvature is only in the transverse coordinates $\tilde{x}$. Choosing conveniently scaled coordinates and replacing $\delta^{2}(\tilde{x})$ by

$$
\frac{1}{\pi \varepsilon^{2}} \theta(\varepsilon-\tilde{r})
$$

where $\tilde{r}=|\tilde{x}|$, we find that for $\tilde{r} \leq \varepsilon$, the transverse components of the metric must be those of a sphere,

$$
\mathrm{d} s^{2}=\mathrm{d} \tilde{r}^{2}+\sin ^{2} \tilde{r} \mathrm{~d} \varphi^{2}+\mathrm{d} z^{2}-\mathrm{d} t^{2}, \quad \tilde{r} \leq \varepsilon .
$$

At $\tilde{r}=\varepsilon$, the transverse metric changes into that of a cone. The cone that touches the sphere at that point generates the metric

$$
\mathrm{d} s^{2}=\mathrm{d} r_{1}^{2}+\left(r_{1} \cos \varepsilon\right)^{2} \mathrm{~d} \varphi^{2}+\mathrm{d} z^{2}-\mathrm{d} t^{2}, \quad r_{1} \cos \varepsilon \geq \sin \varepsilon
$$

where the coordinate $r_{1}$ is matched to the coordinate $\tilde{r}$ at the point $\tilde{r}=\varepsilon, r_{1}=\tan \varepsilon$. The deficit angle $\alpha$ of the cone is $2 \pi(1-\cos \varepsilon)$.

Inside the smeared region, where the metric is that of (2.3), we have the Ricci curvature

$$
R_{x x}=R_{y y}=1, \quad R_{z z}=R_{t t}=0
$$


Fig. 2 (a) Cross section of 3 -space surrounding a positive string, with deficit angle $A A^{\prime}$; (b) Cross section of 3 -space surrounding a negative string, showing a surplus angle $A A^{\prime}$
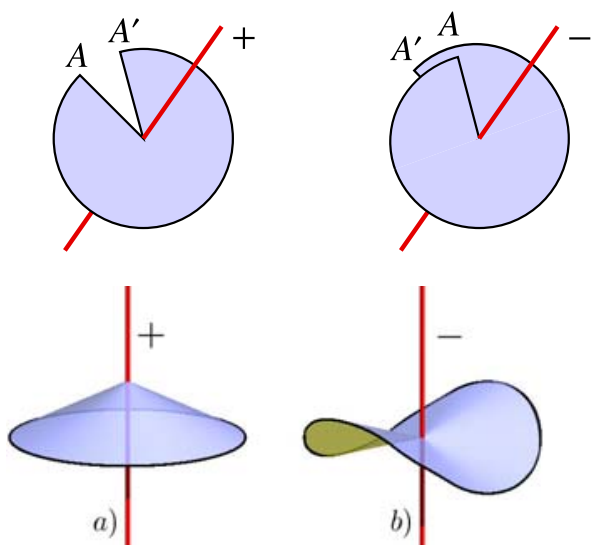

while on the conical metric (2.4), the curvature vanishes. Substituting (2.1) into Einstein's equation, one then gets

$$
8 \pi G \varrho \frac{1}{\pi \varepsilon^{2}}=1,
$$

so that for small values of $\varepsilon$, the deficit angle can be written as

$$
\alpha=2 \pi(1-\cos \varepsilon) 8 G \varrho \varepsilon^{2}=8 \pi G \varrho .
$$

We quickly return to the old coordinates where the delta peak is very sharp. Spacetime surrounding a string is then seen to be as sketched in Fig. 2a. When the string constant $\varrho$ is large, we redefine it to be the one generating exactly the deficit angle $\alpha=8 \pi G \varrho$. Note that a positive string constant leads to a deficit angle. A negative string constant would produce a surplus angle, see Fig. 2b. Normally, in string theory, a negative string constant would make a string highly unstable, as it contains positive pressure and negative energy. In our case, however, strings are constrained to form straight lines, and therefore this instability has no effect at small distances. We later may wish to include such negative strings in our models. We leave this open for the time being, but unless indicated specifically, we will usually be discussing strings with positive string constants and thus with positive deficit angles.

A more conventional derivation of the locally flat metric surrounding a string can be found for instance in [15].

\section{Moving Strings}

A description of a multitude of static strings is now straightforward, in principle. However, already here, one may expect considerable complications. The system of static strings is not unlike the $2+1$ dimensional universe with moving point particles, where the time coordinate is replaced by the coordinate $z$ of the static string system. We know that the $2+1$ dimensional world has either a big bang singularity or a 
big crunch [4, 5]. Similar "infrared" singularities might show up in the static string system. This question will not be further pursued here. It is the local properties of the model that we will investigate further.

Moving strings impose important questions concerning the internal consistency of the model. A moving string can be characterized in different ways. Firstly, one can specify the orientation vector $\vec{\omega}$ of the string, normalized such that its norm coincides with half the deficit angle, $\frac{1}{2} \alpha=4 \pi G \varrho$ when the string is at rest:

$$
|\vec{\omega}|=\frac{1}{2} \alpha .
$$

In addition then we specify the velocity vector $\vec{v}$ of the string. But, noticing that the string is invariant under boosts in the direction $\vec{\omega}$, only the component of $\vec{v}$ orthogonal to $\vec{\omega}$ matters, so we limit ourselves to the case

$$
\vec{v} \cdot \vec{\omega}=0
$$

Finally, the position of the string at $t=0$ should be specified. This requires another vector orthogonal to $\vec{\omega}$. All in all, this requires $3+2+2=7$ real parameters, of which 5 are translationally invariant, and 2 can be set to zero by a spacelike translation in 3-space.

Alternatively, we can specify the string's characteristics by giving the element of the Poincare group that describes the holonomy along a non-contractible cycle $C$ around the string. For static strings through the origin of 3-space, this is just the pure rotation operator, which will be denoted as $U(\vec{\omega})$. For strings moving with velocity $\vec{v}$ through the origin, this is the element $B(\vec{v}) U(\vec{\omega}) B(-\vec{v})$ of the Lorentz group, where $B(\vec{v})$ is the element of $S L(2, C)$ that represents a pure Lorentz boost corresponding to the velocity $\vec{v}$.

If the string does not move through the origin, we get a more general element of the Poincaré group.

Notice however, that an arbitrary element of the Lorentz group is specified by 6 parameters, not 5 , and the elements of the Poincare group by 10 parameters, not 7 . This means that not all elements of the Poincaré group describe the holonomy of a string. Firstly, ignoring the translational part, the pure Lorentz transformation $Q$ associated to the closed curve $C$ has to obey one constraint:

There must be a Lorentz frame such that, in that frame, $Q$ is a pure rotation in 3-space.

Since we plan to describe these Lorentz transformations in terms of their representations in $S L(2, C)$, we identify this as a constraint on the associated $S L(2, C)$ matrices. Write

$$
Q=B(\vec{v}) U(\vec{\omega}) B(-\vec{v}),
$$

where $B(\vec{v})$ are $2 \times 2$ matrices representing boosts with velocity $\vec{v}$ (we will see shortly that pure boosts are represented by Hermitean $2 \times 2$ matrices, (3.20)), and $U$ is a unitary matrix representing a pure rotation. When $\vec{\omega}$ points in the $z$ direction, we 
have

$$
U(\vec{\omega})=\left(\begin{array}{cc}
e^{i \omega} & 0 \\
0 & e^{-i \omega}
\end{array}\right)
$$

so that

$$
\operatorname{Tr}(U)=2 \cos \omega .
$$

Since the trace is invariant under rotations and the boosts (3.3), it follows quite generally that

$$
\begin{gathered}
\operatorname{Im}(\operatorname{Tr}(Q))=0, \\
|\operatorname{Re}(\operatorname{Tr}(Q))| \leq 2 .
\end{gathered}
$$

Equation (3.6) fixes one of the real variables of the Lorentz transformation $Q$. Inequality (3.7) is important in a different way. A generic $S L(2, C)$ matrix can de written in a basis where it is diagonal. Because the determinant is restricted to be 1 , the diagonal form is then

$$
Q=\left(\begin{array}{cc}
z & 0 \\
0 & 1 / z
\end{array}\right),
$$

where $z$ can be any complex number. Imposing (3.6) leaves two options: either $z$ is on the unit circle-in which case it represents a pure rotation in 3-space, or it is a positive or negative real number. In the latter case, $Q$ is a pure Lorentz boost, and this is when (3.7) is violated. It describes the holonomy of something that is quite different from a string. We return to that at the end of this section.

The second restriction to be imposed on the holonomy of a physical string is the translational part of the element of the Poincaré group. We just saw that in the frame where $Q$ is diagonal, the string is static, and its position should be characterized by a vector orthogonal to $\vec{\omega}$. Using the same notation (3.3) to write the full Poincare element $P$, we add a displacement operator $D(\vec{u})$ ( $\vec{u}$ being the displacement vector):

$$
P\left(\alpha_{v}^{\mu}, y^{\mu}\right)=B(\vec{v}) D(\vec{u}) U(\vec{\omega}) D(-\vec{u}) B(-\vec{v}),
$$

where $\alpha_{\nu}^{\mu}$ is the generator of the Lorentz transformation, and $y^{\mu}$ is the 4 dimensional displacement vector:

$$
(P x)^{\mu} \stackrel{\text { def }}{=}(Q x)^{\mu}+y^{\mu} \stackrel{\text { def }}{=} L(\alpha)_{\nu}^{\mu} x^{\nu}+y^{\mu} .
$$

Henceforth, expressions such as $Q x$ stand short for a $4 \times 4$ matrix $L_{v}^{\mu}$ acting on the 4-vector $x^{\mu}$. In $S L(2, C)$ notation, this would read

$$
(Q x)^{0} \mathbb{I}+(Q x)^{a} \sigma_{a}=Q\left(x^{0} \mathbb{I}+x^{a} \sigma_{a}\right) Q^{\dagger},
$$

where $\sigma_{a}$ are the three Pauli matrices. From (3.9), we have

$$
y=B(\vec{v})(u U(\vec{\omega})-U(\vec{\omega}) u) B(-\vec{v})
$$


In the static case, $B=\mathbb{I}, y$ can neither have a time component nor a component parallel to $\vec{\omega}$. The time component could be introduced to describe a spinning string, analogous to a spinning point particle in $2+1$ dimensional gravity, but the problem with that is that such a space-time would possess closed timelike curves (CTC); thus, causality would be a problem.

A component of $y$ in the $\vec{\omega}$ direction could be introduced as a generalization of the string concept; it would describe a string with torsion—one could call that a "spring". We will not discuss springs further, but we have to keep this possibility in mind. Barring spin and torsion, gives two constraints on the vector $y^{\mu}$.

Specifying the element of the Poincare group that describes the holonomy associated to a non-contractible curve around a string, specifies its position provided $\vec{\omega}$ does not vanish (vanishing strings have no specified position). To find the location of a string if $P$ is given is easy: just solve the equation

$$
P x=x .
$$

In the static case, this gives

$$
x=s \vec{\omega}+t e^{0}
$$

where $e^{0}$ is the unit vector in the time direction, while $s$ and $t$ are free parameters. Thus, we find the string world sheet.

We end this section with a few important facts about Lorentz transformations in the $S L(2, C)$ representation, for future use.

(1) Pure rotations in 3-space are described by the subgroup $S U(2)$ of $S L(2, C)$. Thus, $Q$ is a pure rotation iff $Q^{\dagger}=Q^{-1}$. We often write such a matrix as

$$
U(\vec{\omega})=c \mathbb{I}+i \sum_{a} s_{a} \sigma_{a},
$$

where

$$
c=\sqrt{1-\sum_{a} s_{a}^{2}}
$$

and $\sigma_{a}$ are the three Pauli matrices. $s_{a}$ is the rotation vector $\vec{\omega}$ but with a different normalization:

$$
s_{a}=\frac{\sin |\omega|}{|\omega|} \omega_{a} .
$$

For a pure rotation along the $z$-axis, see (3.4), $c=\cos (\omega)$ and $s_{3}=\sin (\omega)$. Note that, as before (3.1), $\omega$ is half the full rotation angle.

(2) Pure Lorentz boosts $B(\vec{v})$ are Hermitean $S L(2, C)$ matrices. Diagonalizing such a matrix corresponds to rotating $\vec{v}$ into the $z$-direction. The matrix then takes the form

$$
B(\vec{v})=\left(\begin{array}{cc}
r & 0 \\
0 & 1 / r
\end{array}\right), \quad v=\frac{r^{2}-1}{r^{2}+1} .
$$


In general,

$$
B(\vec{v})=\operatorname{ch} \mathbb{I}+\sum_{a} s h_{a} \sigma_{a},
$$

where the vector $\overrightarrow{s h}$ is $\vec{v}$ apart from a normalization:

$$
s h_{a}=\frac{v_{a}}{\sqrt{1-v^{2}}}, \quad c h=\frac{1}{\sqrt{1-v^{2}}} .
$$

Notice that $\operatorname{Tr}(B) \geq 2$. Hermitean matrices with $\operatorname{Tr}(B) \leq-2$ are equivalent to $-B$ since all $S L(2, C)$ matrices $Q$ describe the same Lorentz transformation as $-Q$.

(3) Any Lorentz transformation $Q$ can be associated to a vector $\vec{v}$ and a vector $\vec{\omega}$ such that it is the product of a pure boost and a pure rotation:

$$
Q=B(\vec{v}) U(\vec{\omega}) .
$$

Proof: define the matrix $R$ by

$$
R=Q Q^{\dagger}
$$

and notice that, since $R$ is Hermitean and positive definite, it can be written as

$$
R=B^{2},
$$

where $B$ is also hermitian and, $\operatorname{since} \operatorname{det}(Q)=1$, also $\operatorname{det}(B)=1$. Diagonalizing $R$ gives us $B$ in diagonal form, and its eigenvalues (whose product is one), can be matched with the boost velocity $\vec{v}$ which is again in the $z$-direction in this frame. Finally, define $U$ as

$$
U=B^{-1} Q, \quad U U^{\dagger}=B^{-1} Q Q^{\dagger} B^{-1}=\mathbb{I} .
$$

$U$ is unitary, therefore it describes a pure rotation in 3-space.

(4) Given any Lorentz transformation $Q$ with $\operatorname{Im}(\operatorname{Tr}(Q))=0$, then there exists a pure boost operator $B(\vec{v})$ and either a rotation $U(\vec{\omega})$ or another pure boost $B\left(\overrightarrow{v^{\prime}}\right)$ such that

$$
\text { either } \quad Q=B(\vec{v}) U(\vec{\omega}) B(-\vec{v}), \quad \text { or } \quad Q= \pm B(\vec{v}) B\left(\vec{v}^{\prime}\right) B(-\vec{v}) \text {. }
$$

Proof: looking at the eigenvalues of $Q$, one finds that, since their product is one and the sum is real, they either match the eigenvalues of $U(\vec{\omega})$ or those of $\pm B\left(\overrightarrow{v^{\prime}}\right)$. A matrix that diagonalizes $Q$ can be written as $B(\vec{v}) U\left(\vec{\omega}_{1}\right)$ due to the previous theorem. So we have

$$
Q=B(\vec{v}) U\left(\vec{\omega}_{1}\right) R U^{-1}\left(\vec{\omega}_{1}\right) B^{-1}(\vec{v})
$$

where $R$ stands for the matrix that is either a rotation or a boost. This rotation or boost was in the $z$-direction, since $R$ was diagonal. The matrix $U\left(\vec{\omega}_{1}\right)$ rotates that vector into any other direction in 3-space. 
We now return to (3.13) and (3.14) for the string world sheet. Suppose we have a $Q$ that obeys (3.6) but not the inequality (3.7). Then, in some Lorentz frame, this is a pure boost rather than a pure rotation. Write it as

$$
Q=\left(\begin{array}{cc}
r & 0 \\
0 & 1 / r
\end{array}\right),
$$

where we took the boost to be in the $z$-direction. The equation for the "world sheet", $Q x=x$, now leads to

$$
z=0, \quad t=0,
$$

in other words, the transverse plane at $t=0$. This is a spacelike surface rather than a timelike string world sheet. What we find here is a "tachyonic" string. Such elements will be difficult to incorporate in a viable gravity model, if we wish to have some version of causality. We will therefore attempt to avoid structures for which the holonomy is a boost.

\section{Connecting Strings}

We could try to limit ourselves to having only infinite, straight strings in our model, but as soon as collisions are considered — and we will argue that these are inevitableone must face the presence of strings with finite lengths. These strings must then be connected to other strings in junctions, see Fig. 3. Just as our infinite strings, junctions are also surrounded by flat space [15]. The rules for connecting three strings $A, B$ and $C$ are as follows.

(i) The junction at time $t$ must lie on a point $x(t)$ that is on the world sheet of the three strings and is a straight line. Thus, the three world sheets must have a straight line in 4-space in common. This line is a solution of

$$
Q_{A} x=Q_{B} x=Q_{C} x=x
$$

(which is, again, in the 4 dimensional notation). Depending on whether this line is timelike, spacelike or lightlike, there are three classes of junctions: subluminal, superluminal and lightlike. Superluminal junctions will be seen to come in two types.

(ii) The fact that the surrounding space-time is flat implies that the holonomies must match: $Q_{A}=Q_{B} Q_{C}$ or, if we take all strings pointing towards the junction (so that $Q_{A}$ turns into its inverse),

$$
Q_{A} Q_{B} Q_{C}=\mathbb{I} .
$$

Fig. 3 Junction connecting three strings. The holonomy $C_{A}$ of string $A$ equals the product $C_{B} C_{C}$ of the holonomies of strings $B$ and $C$

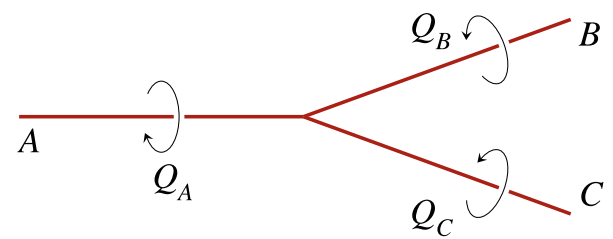


In the case of a superluminal junction, the three strings have a spacelike line in common. This means that a special Lorentz frame exists where this is a straight line in the $z$-direction that is instantaneous in time. The three connecting strings are parallel in that frame, but they may have different velocities. We either have one string splitting in two, or two strings merging into one. The first of these cases is impossible to reconcile with local causality, but the latter, in principle, is: two parallel strings meet and subsequently merge. Since the time reverse of this event violates causality, this would be an example of information loss. At first we will find that this kind of events may be difficult to avoid, but we will show how this can nevertheless be achieved if we so wish.

In more general Lorentz frames, superluminal junctions can be easily recognized as they describe a pair of strings opening up or closing like a superluminal zipper. A superluminal zipper that is opening up will have to be avoided at all times; the closing (joining) superluminal zipper is a curious case of information loss.

Lightlike and timelike (subluminal) junctions are fine.

If we wish two strings $A$ and $B$ to meet at one subluminal junction for an extended amount of time, then this gives three restrictions on the associated holonomies $Q_{A}$ and $Q_{B}$ alone: first, the product of their holonomies must again be a string holonomy, or

$$
\operatorname{Im}\left(\operatorname{Tr}\left(Q_{A} Q_{B}\right)\right)=0, \quad\left|\operatorname{Re}\left(\operatorname{Tr}\left(Q_{A} Q_{B}\right)\right)\right| \leq 2 .
$$

From this, one can show that in a Lorentz frame where string $A$ is static and pointing in the $z$-direction, we have

$$
Q_{A}=\left(\begin{array}{cc}
e^{i \omega} & 0 \\
0 & e^{-i \omega}
\end{array}\right), \quad Q_{B}=\left(\begin{array}{cc}
a_{1}+i a_{2} & b_{1}+i b_{2} \\
\mu\left(-b_{1}+i b_{2}\right) & a_{1}-i a_{2}
\end{array}\right),
$$

where all coefficients are real. Our second restriction now is that, in (4.4),

$$
\mu>0
$$

which corresponds to a subluminal junction. If $\mu<0$ we have a superluminal junction. If $\mu=1$ the string $B$ is static as well. One easily checks that then $Q_{B}$ is unitary and hence a pure rotation. The case for general positive $\mu$ is obtained by Lorentz boosting in the only allowed direction, the $z$-direction (otherwise, $A$ would not remain static). Note that such a boost is described by (3.27).

Finally, of course, the displacement vectors of the Poincaré group elements must also match.

Thus, we will be specially interested in the case where, for every string junction, there exists a Lorentz frame where all three strings are static. If the string constants are large, so that the deficit angles (or possible surplus angles) are large, the situation is a bit complicated, since at a junction the three strings appear not to lie in a single plane. If the string constants are weak, one discovers that, in principle, there are two types of subluminal junctions. They are sketched in Fig. 4. In the first case, see Fig. 4a, either all deficit angles are positive or they are all negative (i.e., all surplus angles are positive). This we will refer to as a regular junction. The strings behave as elastic bands connected at a point: each string appears to pull the two others towards 


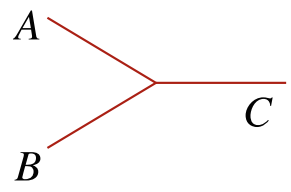

a)

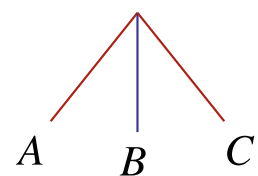

b)

Fig. 4 Two types of string junctions. In case (a), there is always an obtuse angle present. This is the case when all three strings are positive or all three are negative. In case (b), one of the strings, in fact string \# $B$, has a sign opposite to the sign that the other two have in common

Fig. 5 Six string segments connected into a triangle, forming a 'localized' particle

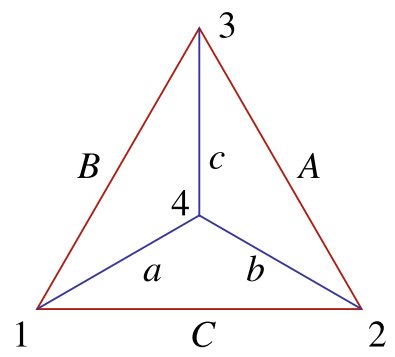

it. In the case one string has a sign opposite to the two others, one gets the situation sketched in Fig. 4b: it is the situation that can be deduced from the previous case by replacing the one string with the exceptional sign by an opposite-sign string pointing in the opposite direction.

This information is useful if one wants to investigate whether constructions can be made with only finite extensions in space. Figure 5 shows an example of this. We have three strings, $A, B$ and $C$ forming a triangle, and three others, $a, b$ and $c$ that connect the three points to a point in the middle. The junctions 1,2 and 3 are irregular because they contain only sharp angles. Clearly, the strings $a, b$ and $c$ must have signs opposite to the signs of $A, B$ and $C$. Junction number 4 is a regular one.

In general, it is easy to argue that finite size constructions with only positive sign strings cannot be possible, since the entire thing is surrounded by flat space; hence there is no gravitational field; the total energy must be zero. This will not be possible with positive energy strings.

Much of the above remains true when the string constants, apart from their signs, are large, but things then are a bit more difficult to visualize, since space and spacetime are locally but not globally flat.

In this paper we will not attempt to completely avoid the emergence of negative string constants. This would lead to negative energy states. One could think of addressing these at a later stage in a quantum theory by some kind of second quantization. As long as we restrict ourselves to local behavior this might not be a disaster, but of course the question of positive and negative string constants (deficit angles) will have to be addressed. We will advocate to avoid superluminal junctions of the splitting type at any stage, as these are difficult to reconcile with causality. Avoiding superluminal junctions of the joining type will be a bit harder, but we will finally find a procedure to avoid those together with the variety that opens up. Also all strings that 


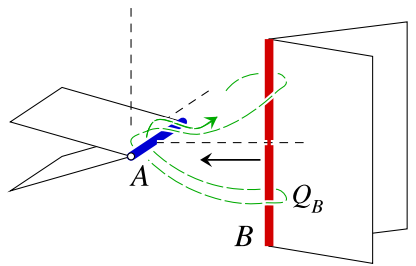

a)

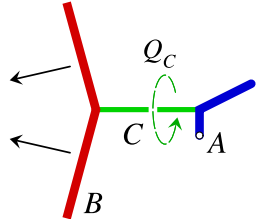

b)

Fig. 6 Orthogonal strings scattering. (a) Initial state. String $B$ moves towards string $A$ (arrow). The cusps caused by their deficit angles are shown. Dashed lines are an orthogonal frame shown for reference. (b) After the scattering, a new string $C$ connecting the first two emerges. Strings $A$ and $B$ now both show a kink. The cusps in the last situation are not shown. The oriented dashed curves explain (5.1)

violate the inequality (3.7) must be avoided since they too are impossible to reconcile with causality. These two demands will require so much of our attention that we will not further dwell on the signs of the string constants.

\section{Orthogonal Collisions}

When we were dealing with point particles, in the $2+1$ dimensional case, we could safely assume that the particles will never collide head-on. In general, they will miss one another, and consequently no further dynamical rules are needed to determine how an $N$ particle system will evolve. This will not be true in higher dimensional spaces. ${ }^{2}$ Strings in $3+1$ dimensional space-time will in general not be able to avoid one another. They will cross, and in doing so, two straight string sections will not be straight anymore after the collision.

Consider an initial state in which two strings are heading towards one another. We can always work in a Lorentz frame where one of the strings, call it $A$, is at rest. The conical 3-space surrounding it has a deficit angle $\alpha=2 \omega_{A}$. In the generic case, in this Lorentz frame, the second string does not have to be oriented orthogonally to the first one. Its string constant, $\beta$, does not have to be the same as $\alpha$. Consider now the velocity vector $\vec{v}$ of the second string. If it is not orthogonal to the string $A$, we perform a Lorentz boost in that direction. String $A$ will stay at rest. If $\vec{v}$ is not orthogonal to the string $B$, we replace it by one that is orthogonal to $B$. This way, one convinces oneself that, in general, we can limit ourselves to the case where $\vec{v}$ is orthogonal to both $A$ and $B$.

However, it is a physical limitation if we also assume $A$ to be orthogonal to $B$. Just because it is special, we consider this case first. The collision event is sketched in Fig. 6. In Fig. 6a, the two strings approach one another. They both drag a space-time cusp with them. Now, what happens when $B$ hits $A$, is best understood by drawing the cusp of $A$ in the opposite direction. The result of that, however, is that string $B$ is seen to have a kink. The same thing happens to string $A$ itself; it develops a kink due

\footnotetext{
${ }^{2}$ Strings will in general not collide head-on in a space-time of more than 4 dimensions. However, the generalizations of the objects we discuss in this paper, in higher dimensions will be branes, not strings.
} 
to the cusp of $B$. After the passage, the two kinks must be connected by a new string, $C$ that stretches with $A$ and $B$ now moving away from one another.

Indeed, we see that, in general, the holonomy of string $C$ is non-trivial; it is obtained from the holonomies $Q_{A}$ and $Q_{B}$ of strings $A$ and $B$ as follows (depending on sign conventions for $Q_{A}, Q_{B}$ and $Q_{C}$ ):

$$
Q_{C}=Q_{B}^{-1} Q_{A}^{-1} Q_{B} Q_{A} .
$$

$Q_{A}$ and $Q_{B}$ do not commute because they represent rotations along two different axes. Clearly, upon crossing, two strings produce a third stretching between them. This is why our model should not be thought of as being globally finite. Every crossing produces more new string segments, so that, in the absence of possible quantum effects, any regular but non-trivial initial condition will eventually create states in which myriads of tiny string segments cover all of space-time. If the original defect angles were relatively small, their commutators will be again much tinier, so the newly created strings are very weak ones. Locally, however, we still have straight string segments surrounded by flat space-time.

There is an important remark to be made here. If the original strings have been approaching each other with velocities close to that of light then the orthogonal velocities will also be close to that of light after the collision. However, then we can easily run into the situation that the newly produced junctions will go faster than light: they will be superluminal. Since they will be of the "joining" variety, these junctions will not violate causality.

Our strategy will be to search for models where the total set of possible string holonomies is a finite one, or else at least discrete, but this we leave for later investigations. There is a more urgent problem that we have to face first.

\section{Slanted Collisions}

In the previous section the result was explained of a collision between two strings and a relative velocity vector that are all orthogonal. What happens when the angles have different values?

In this case, one can convince oneself that no solution is possible with a single string stretching between the outgoing strings. This can be understood by studying the geometry, as sketched in Fig. 7. But we can also verify that, in general, the holonomy (5.1) is not of the string type: it violates (3.6).

To save the model, one can now propose the following. When two strings $A$ and $B$ collide at an angle $\varphi \neq 90^{\circ}$, not one but two new strings appear, ${ }^{3}$ both stretching from $A$ to $B$. A single string cannot be associated with a holonomy of the form (5.1), but a pair of strings can. The question is now, whether the data of this pair of strings would be uniquely determined by the initial characteristics of $A$ and $B$. To investigate this question, the author combined analytical arguments with computer calculations, just to see how things will work out. The topology is defined in Fig. 8.

\footnotetext{
${ }^{3}$ Later, in Sect. 8, we will see that even more than two new strings may emerge.
} 
Fig. 7 Scattering at an angle, $\varphi \neq 90^{\circ}$. The cusp of string $A$ is shown, and the effect it has on string $B$. After the collision, if the cusp would be kept open, these two parts would still form a straight line. Closing the cusp would move point $P$ to point $Q$. If at $P$ the string touches the cylinder surrounding $A$, it should do this now at point $Q$. We see that then $B_{1}$ and $B_{2}$ do not intersect

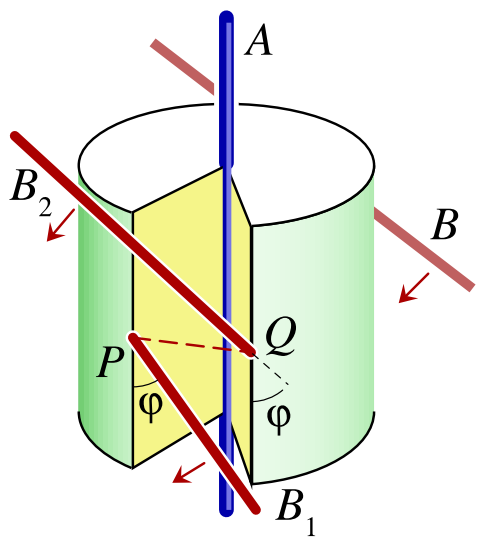

Fig. 8 Scattering at an angle produces two new strands. (a) $A$ and $B$ enter. (b) Since $A$ passes the cusp of $B$ and vice versa, strands $A_{2}$ and $A_{3}$ form angles. These four pieces are labelled 1-4. Four new string pieces must be further specified, here labelled $a-d$

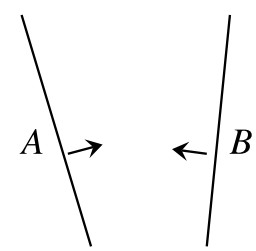

a)

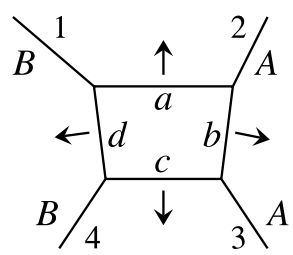

b)

At first sight, it seems that we have considerable freedom to define the orientations, strengths and velocities of the 'internal' strings $a-d$. However, if we fix one of these, all others are determined since the holonomies at a junction must obey (4.2). In addition, the strings must be properly attached to one another. As it turns out, the matching of the strings is guaranteed if (4.2) is obeyed at all junctions, and if in addition the string conditions (3.6) and (3.7) are obeyed by all four new holonomies, $a-d$.

The holonomy matrices at the 'external lines' $1-4$ are fixed by the initial conditions. Originally, we had, in one conveniently chosen Lorentz frame, $Q_{1}=Q_{B}$ and $Q_{3}=Q_{A}^{-1}$. Here, the inverse sign arises if we decide to consider the holonomies with respect to observers looking towards the interaction point, and the holonomy curves $C$ are chosen to go clockwise. Then,

$$
\begin{aligned}
& Q_{4}=Q_{3}^{-1} Q_{1}^{-1} Q_{3}, \\
& Q_{2}=Q_{1}^{-1} Q_{3}^{-1} Q_{1} .
\end{aligned}
$$

The last equation is actually the one required for consistency with the demand that

$$
Q_{1} Q_{2} Q_{3} Q_{4}=\mathbb{I} .
$$

Given the holonomy $Q_{a}$ of the string section $a$, the others can be defined as follows:

$$
Q_{b}=Q_{a} Q_{2}, \quad Q_{c}=Q_{b} Q_{3}, \quad Q_{d}=Q_{a} Q_{1}^{-1},
$$


which is the most systematic definition, and consistency with (6.2) is ensured. We emphasize that there is always some ambiguity in defining the Lorentz frames for the holonomies $Q_{1}-Q_{4}$ and $Q_{a}-Q_{d}$, so we use (6.1)-(6.3) also to specify these frames.

Let now all external holonomies $Q_{1}-Q_{4}$ be given. How much freedom is there for $Q_{a}-Q_{d}$ ? We do not need to consider the translation parameters in the Poincaré group; these will be taken care of automatically, since there is a point $(0,0,0,0)$ where the colliding strings $A$ and $B$ first met. This point can be kept at the origin of our coordinate frame. Thus, we consider the equations for the elements of the Lorentz group, which are most conveniently described as $S L(2, C)$ matrices. Each element of the Lorentz group is characterized by 6 real variables: a rotation vector and a velocity vector, or alternatively the four complex numbers in a $2 \times 2$ matrix $Q$, subject to the constraint that the complex number $\operatorname{det}(Q)$ should be set equal to 1 .

The junction equations (6.1)-(6.3) leave us the freedom to choose $Q_{a}$. This gives a space with 6 real parameters. Then the string equation (3.6) for $Q_{a}-Q_{d}$, together gives us 4 real constraints. The surviving 2-dimensional manifold is then further constrained by the demands (3.7). Thus, the manifold of all possibilities is a twodimensional space.

To obtain somewhat more understanding of this manifold, let us consider the 8 dimensional set of all $L(2, C)$ matrices for $Q_{a}$, without the nonlinear constraint concerning the determinant. The conditions (3.6), $\operatorname{Im} \operatorname{Tr}\left(Q_{a, b, c, d}\right)=0$, in combination with the junction equations (6.3), are 4 linear equations for the matrix elements of $Q_{a}$. This leaves us with a linear $8-4=4$ dimensional space. Then we have the inequalities (3.7), which for the matrix $Q_{a}$ imply that

$$
Q_{a}=\left(\begin{array}{ll}
a_{1}+i a_{2} & b_{1}+i b_{2} \\
c_{1}+i c_{2} & d_{1}-i a_{2}
\end{array}\right), \quad\left|a_{1}+d_{1}\right| \leq 2,
$$

and similarly for the three other internal holonomies. Realizing that, in our 4 dimensional space, these conditions can be written as

$$
\left|e_{i} \cdot x\right| \leq 2, \quad i=1, \ldots, 4
$$

and assuming that, in general, the four vectors $e_{i}$ will be independent, we see that, in the generic case, the surviving space is a compact one: a four dimensional hypercube. We can be sure that the inequalities (6.5) give us a non empty four dimensional space.

Next, however, we have the two constraints

$$
\operatorname{Re}\left(\operatorname{det}\left(Q_{a}\right)\right)=1, \quad \operatorname{Im}\left(\operatorname{det}\left(Q_{a}\right)\right)=0 .
$$

These two equations for $Q_{a}$ ensure that the same equation will hold for $Q_{b}-Q_{d}$, because the determinant is preserved, and because $\operatorname{det}\left(Q_{i}\right)=1$ also for the external $Q_{1}-Q_{4}$. Now these are quadratic equations for the coefficients of $Q_{a}$, so the question whether these two equations are compatible with the inequalities (6.5) and with one another is a more delicate one. It can be simplified in the following way.

First, we can sit in a frame where string $B$ is stationary and oriented in the $z-$ direction, or more precisely,

$$
Q_{B}=Q_{1}=\left(\begin{array}{cc}
e^{i \omega} & 0 \\
0 & e^{-i \omega}
\end{array}\right) .
$$


In that case, the condition $\operatorname{Im}\left(\operatorname{Tr}\left(Q_{d}\right)\right)=0, Q_{d}=Q_{a} Q_{1}^{-1}$, see (6.3) and (3.6), implies that the coefficients for $Q_{a}$ in (6.4) obey

$$
\operatorname{Im}\left(e^{-i \omega}\left(a_{1}+i a_{2}\right)+e^{i \omega}\left(d_{1}-i a_{2}\right)\right)=0 \quad \longrightarrow \quad d_{1}=a_{1} .
$$

Therefore, we can write

$$
Q_{a}=\left(\begin{array}{cc}
a & b \\
c & a^{*}
\end{array}\right),
$$

where $a, b$ and $c$ are complex numbers. The condition that $\operatorname{det}\left(Q_{a}\right)$ is real can now be written as

$$
c=-\mu_{1} b^{*},
$$

where $\mu_{1}$ is a real parameter. This is (4.4). We will usually limit ourselves to the case $\mu_{1}>0$, the junction with $Q_{1}$ is then subluminal. The condition that the real part of the determinant is 1 can now be written as follows:

$$
\begin{aligned}
Q_{a} & =\lambda\left(\begin{array}{cc}
1+i a_{2} & b_{1}+i b_{2} \\
\mu_{1}\left(-b_{1}+i b_{2}\right) & 1-i a_{2}
\end{array}\right), \quad \mu_{1}, a_{2}, b_{1}, b_{2} \text { real, } \\
\lambda & =1 / \sqrt{1+a_{2}^{2}+\mu_{1}\left(b_{1}^{2}+b_{2}^{2}\right)} .
\end{aligned}
$$

Note that choosing $\mu_{1}>0$ ensures that the square root is real.

The condition that the traces of $Q_{b}$ and $Q_{c}$ are real form two linear conditions on the three coefficients $a_{2}, b_{1}$ and $b_{2}$ (where only the parameter $\mu_{1}$ appears non linearly). Suppose that these are used to fix $b_{1}$ and $b_{2}$. Then we are left with $\mu_{1}$ and $a_{2}$ as two independent free parameters.

The question that remains is whether we can also obey the inequalities (3.7) for the string holonomies $Q_{a}-Q_{d}$. Those for $Q_{a}$ and $Q_{d}$ can easily be read off:

$$
\begin{array}{ll}
Q_{a}: & |\lambda| \leq 1 \quad \longrightarrow \quad a_{2}^{2} \geq-\mu_{1}\left(b_{1}^{2}+b_{2}^{2}\right), \\
Q_{d}: \quad\left(\sin \omega-a_{2} \cos \omega\right)^{2} \geq-\mu_{1}\left(b_{1}^{2}+b_{2}^{2}\right),
\end{array}
$$

which is ensured if we choose $\mu_{1}>0$.

Next, we can perform the same trick at the junction with $Q_{2}$ or at the junction $Q_{3}$. If we choose $Q_{2}$ then only one further linear constraint on the coefficients $a_{i}$ and $b_{i}$ follows, and so we have two freely adjustable parameters $\mu_{1}$ and $\mu_{2}$ that now parameterize our two-dimensional manifold. We may freely limit ourselves to positive values of $\mu_{1}$ and $\mu_{2}$ so that we can be sure that the junctions connecting $Q_{1}$ and $Q_{2}$ to the quadrangle are both subluminal. Unfortunately however, this gives us no guarantee that $Q_{3}$ and $Q_{4}$ will be subluminal as well, and the line joining them, string segment $Q_{c}$, is then not guaranteed to obey the necessary inequality (3.7) that would ensure it to be a subluminal string.

There is a smarter way to proceed: we pick two opposite junctions, say $Q_{1}$ and $Q_{3}$. Now, however, our numerical calculations show us a surprise. At least this author had not expected the special thing that happens. 
Suppose we first go to the Lorentz frame where $Q_{a}$ and $Q_{d}$ are static. Choose $Q_{1}$ to be a rotation along in the $z$-axis. Then $Q_{a}$ and $Q_{d}$ are both described by the parametrization of (6.11), with freely adjustable $\mu_{1}$. The coefficients in this frame obey four real constraints, but since the determinant is known to be real, these are actually just three new, linear constraints.

Now perform the Lorentz transformation that makes $Q_{3}$ a static rotation along the $z$-axis. Again assume a freely adjustable parameter $\mu_{3}$ and four constraints on the coefficients, of which only three are independent because of the determinant. One would have thought to end up with all coefficients fixed, apart from the two freely adjustable parameters $\mu_{1}$ and $\mu_{3}$.

But this is not what happens. The four linear constraints on the parameters are independent, while, instead, the two parameters $\mu_{1}$ and $\mu_{3}$ are not independent. They are found always to be related by an equation of the form

$$
\mu_{3}=\frac{A+B \mu_{1}}{C+D \mu_{1}},
$$

where the coefficients $A, B, C$ and $D$ depend in a complicated way on the data that describe the holonomies of the external lines only. This is true whenever the holonomies $Q_{1}$ and $Q_{3}$ obey the string equation (3.6).

This puts our problem in a different perspective: we can only succeed in devising an acceptable pattern of a single quadrangular string loop if the coefficients $A, B, C$ and $D$ allow for positive values for both $\mu_{1}$ and $\mu_{3}$. Conversely, if we have such a solution then we are guaranteed that all four internal strings are Lorentz transformations of static ones, and hence they all obey the inequality (3.7). However, we found that the coefficients can obtain all sorts of values. It is possible that $A$ and $B$ are negative while $C$ and $D$ are positive. In that case, it may well be that no acceptable solution exists. We return to this case in the next section.

Since $\mu_{1}$ and $\mu_{3}$ are not independent, they only fix one parameter of our two dimensional manifold. We can now return to introducing $\mu_{2}$ as the other parameter. The relation between $\mu_{2}$ and $\mu_{4}$ is similar to the one between $\mu_{1}$ and $\mu_{3}$. So, again, we have four coefficients $A, B, C$ and $D$, of which we must check whether they allow two positive values for $\mu_{2}$ and $\mu_{4}$. If so, we have a solution with only subluminal junctions and subluminal strings.

The explicit expressions for the four coefficients are too lengthy to be displayed here. We checked numerically that indeed $\mu_{1}$ and $\mu_{2}$ are independent, so together they can be used to search a suitable point of our two-parameter space. The resulting relations between the coefficients $a_{i}$ and $b_{i}$ now completely determine their values.

We checked explicitly with numerical examples that the above procedure appears to work flawlessly. If the two sets of coefficients $A-D$ allow for positive $\mu$ values, $\left(\mu_{1}, \ldots, \mu_{4}>0\right)$ this guarantees that all internal lines obey the string equation (3.6), that the four junctions at $Q_{1}-Q_{4}$ are all subluminal, and that the four strings $Q_{a}-Q_{d}$ also obey the string inequality (3.7).

However, we have to check explicitly the existence of two positive $\mu$ values. In (6.14), with $C$ normalized to one, two positive (or vanishing) values are excluded 
only if ${ }^{4}$

$$
A<0, \quad B<0, \quad C=1, \quad D>0 .
$$

Thus, we have to exclude this domain for the two sets of antipodal points. It was found however, that this domain can actually easily be entered, when the external holonomy operators $Q_{1}-Q_{4}$ are far from the identity. So, if that happens, we have no one-string-loop solution with the given topology.

A simple symmetry argument (see Appendix) shows that the coefficients $A-D$ relating $\mu_{4}$ to $\mu_{2}$ are a simple permutation of the coefficients relating $\mu_{3}$ to $\mu_{1}$. It follows that, if there are positive solutions for $\mu_{1}$ and $\mu_{3}$ then also positive solutions exist for $\mu_{2}$ and $\mu_{4}$.

Note however, that we can also choose the crossed diagrams. As in the Feynman diagrams of quantum field theory, we have besides the original loop two crossed diagrams, such as the one obtained by interchanging the points 2 and 3 . Each of these can be tried, but still there is no guarantee that a solution of this form will always exist.

Finally, there is another important question to ask: will the internal holonomies $Q_{a}-Q_{d}$ all describe string sections with positive string constants (positive defect angles)? To check this is technically awkward. It means that negative energy strings are not excluded for the time being. They are not as harmful as the strings that violate causality, but still, one might prefer to have only states with positive local energy densities. It seems that the wrong sign can easily come up. We decide to postpone this question.

In fact, the orthogonal scattering case, described in Sect. 5, would generate superluminal junctions unless we replace the solution by our double string diagram. Here however, superluminal junctions seem to be impossible to avoid unless we allow some of the internal string sections to have the wrong sign for their string constants. The sign problem, therefore, appears to be difficult to avoid.

\section{Other Transitions}

In the previous section it was found that there are two regions defined by the inequalities (6.15) (one for each diagonal), that we have to stay out of. The regions are exclusively defined by the external holonomy matrices $Q_{1}-Q_{4}$, that is, by the initial string configuration. So if we enter any one of these regions, the result of this collision cannot be the configuration sketched in Fig. 7. Therefore, in that case, we have to try something else. To do this, we made a further study of the coefficients $A-D$. It was found that they enforce $\mu_{1} \approx \mu_{3} \approx 1$ when the relative velocities of the external strings are all non-relativistic. This is the allowed region. What if strings collide relativistically?

\footnotetext{
${ }^{4}$ The case where one or more of these coefficients are equal to zero might be admissible, since lightlike joints do not seem to violate causality.
} 
Fig. 9 Scattering producing a multitude of new string sections, investigated in the text

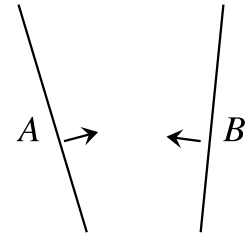

a) b)

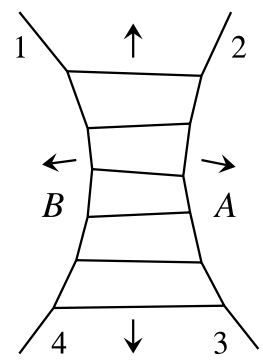

We checked the case where $Q_{1}$ and $Q_{2}$ are large, with possibly relativistic relative velocities, but

$$
E \stackrel{\text { def }}{=} Q_{2} Q_{3}=\left(Q_{4} Q_{1}\right)^{-1} \approx \mathbb{I} .
$$

If this case could be handled, then we can try more complicated scattering diagrams, of the kind depicted in Fig. 9. If we consider a sufficiently large number of intermediate strands in this collision process, condition (7.1) can be realized in all subsegments. This would guarantee the possibility of this multiple strand final state if the state with just two strands would be forbidden. Even this, however, is difficult to prove. If the external holonomies obey (7.1) then the coefficients $A-D$ linking diagonally opposite junctions depend entirely on the details of the matrix elements of $E$, regardless how close this matrix is to the identity, as explicit algebraic calculations show. From this it follows that both the allowed and the forbidden domains touch the point $E=\mathbb{I}$. We were not yet able to prove that configurations with either a single internal quadrangle or multiple strands suffice to cover all eventualities, as the space of all possible external holonomies $Q_{1}-Q_{4}$ is very large.

Strings crossing over is not the only kind of "events" that can take place in this model. We can also encounter the situation where a string bit, of the kind that results from collisions of the type described in the above, is reduced to zero length. It is bounded by two other junctions that herewith merge into one. Our first try should be whether the result could again be a one-strand, two-strand, or multiple strand final state, just like the ones described earlier. However, if we allow ourselves strings with negative string constants, then there is a simpler final state: the one where the original string gets a "negative length". This is really a string where the deficit angle has switched sign. Once it was decided to allow their presence, we could allow them here as well.

\section{Discussion and Conclusions}

We conclude that it may well be possible to construct a complete model for classical (i.e. unquantized) General Relativity with matter, which allows for the construction of piecewise exact solutions in space-time. The model consists exclusively of piecewise straight string segments, surrounded by locally flat regions of Minkowski space-time. This means that these string segments actually also encompass the gravitational degrees of freedom. Interaction occurs when two pieces of string intersect, or when the 
length of one or more string pieces shrinks to zero. At every intersection, at least three (in the case of orthogonal scattering), but nearly always at least six new string sections appear (described by the four finite segments in the quadrangle of Fig. 8, and remembering that the two original strings each split in two). In the latter case, the properties of these new string segments, their string constants, as well as their orientations and velocities, are all described by a point in a compact two parameter space. These freely adjustable points at every interaction junction in 4-space correspond to the freedom one has in choosing the matter interactions. This is not obviously an Euler-Lagrange system, since it cannot be mapped onto its time-reverse. Indeed, one expects that, as time evolves, the string segments become smaller and smaller, and more numerous as well. Also, the two-dimensional parameter space at each intersection is too small to allow us to choose the 4 new string constants from a discrete set of a priori possibilities, and therefore, the string constant parameter space will form a continuum, unlike what one would expect in a realistic model of the real world.

The above are enough reasons why we will not advocate "quantization" of this model along the usual procedures. Quantization will have to go by means of the "prequantization" procedure proposed earlier [16]. This however will require some further refinements that will be explained in a separate paper. The reason why we keep the subject of quantization separate is that it requires basically new assumptions, and that the model described here could be used for different purposes.

There are quite a few open questions apart from quantization. First of all, one would like the model to be complete, that is, give a well formulated prescription under all circumstances how the evolution evolves. We found that many but not all pairs of strings, upon intersecting, can evolve exactly as shown in Fig. 8. When the relative velocities upon impact are high and the string constants are large, more than four new strands may have to appear. One might even suspect an instability such as the formation of a black hole horizon, although precisely in this model one might also suspect the converse, that black holes cannot form. If all string constants are kept positive, localized matter configurations cannot exist, whereas all gravitational curvature must be associated with strings- there is no pure gravity in this model. So, if there is a black hole, strings will have to stick out from it.

The absence of pure gravity degrees of freedom is intriguing. In a sense, matter here is "unified" with gravity, not, as in many models, because gravity generates particle-like degrees of freedom, but the converse, because the matter degrees of freedom, here the string bits, carry around all the space-time curvature there is.

It appears that one might have to decide also to allow for "negative" strings, featuring surplus angles rather than deficit angles. The question must be answered whether or not our newly formed string segments can always be arranged such that they will all be positive ones. Judging from Fig. 4b, this is unlikely but perhaps not impossible.

Also an important question is how to describe our choice for a point in parameter space at every intersection. Parameter space is compact, but the space of all possible collisions is not. After accounting for all symmetries such as the Poincare group at the center of mass, we are left with a non-compact 4 dimensional space of all possible collision parameters. We need an infinite dictionary to describe the parameters for what happens at all these possible interactions. As we had to discover, this space is too large to exclude the existence of corners where further complications arise. 
Apart from all such questions, the model described here might be quite useful to address all sorts of conceptual questions in classical and quantum gravity. The one thing it does not suffer from is ultra-violet divergences, although the infrared question (the question as to what happens at large distances and time intervals) will be quite difficult. Strings could terminate in infinitely dense fractals of string segments, where they could close the universe.

Acknowledgement The author thanks K. Sfetsos for a discussion of this work.

Open Access This article is distributed under the terms of the Creative Commons Attribution Noncommercial License which permits any noncommercial use, distribution, and reproduction in any medium, provided the original author(s) and source are credited.

\section{Appendix: The Algorithm for a Quadrangle Configuration}

In Fig. 8b, we define the holonomies of the external lines to be $Q_{1,2,3,4}$, obeying

$$
Q_{1} Q_{2} Q_{3} Q_{4}=\mathbb{I} .
$$

The internal lines have $Q_{a, b, c, d}$ with

$$
Q_{a}=Q_{d} Q_{1}, \quad Q_{b}=Q_{a} Q_{2}, \quad Q_{c}=Q_{b} Q_{3}, \quad Q_{d}=Q_{c} Q_{4} .
$$

To do the calculations, we avoid square roots by setting

$$
Q_{2}=\Omega_{2}, \quad Q_{1}=Q_{v} \Omega_{1} Q_{v}^{-1}, \quad \Omega_{i}=\left(\begin{array}{cc}
\frac{1+i t_{i}}{1-i t_{i}} & 0 \\
0 & \frac{1-i t_{i}}{1+i t_{i}}
\end{array}\right),
$$

where

$$
Q_{v}=\frac{1}{\left(1-v^{2}\right)\left(1+w^{2}\right)}\left(\begin{array}{cc}
1+v^{2} & 2 v \\
2 v & 1+v^{2}
\end{array}\right)\left(\begin{array}{cc}
1-w^{2} & 2 w i \\
2 w i & 1-w^{2}
\end{array}\right) .
$$

Furthermore,

$$
Q_{3}=Q_{1} Q_{2}^{-1} Q_{1}^{-1}, \quad Q_{4}=Q_{1} Q_{2} Q_{1}^{-1} Q_{2}^{-1} Q_{1}^{-1} .
$$

All $Q$ 's obey

$$
\operatorname{Im} \operatorname{Tr}(Q)=0, \quad|\operatorname{Re} \operatorname{Tr}(Q)|<2 .
$$

The internal strings are then parameterized as follows:

$$
Q_{a}=\lambda\left(\begin{array}{cc}
1+i y_{11} & x_{12}+i y_{12} \\
-\mu_{2}\left(x_{12}+i y_{12}\right) & 1-i y_{11}
\end{array}\right)
$$

where $\lambda$ will be adjusted such that $\operatorname{det}\left(Q_{a}\right)=1$. Together with (A.2), this specifies all string parameters. We now define the holonomies $Q_{e, f, g, h}$ as being the original internal holonomies $Q_{a, b, c, d}$ in the basis where $Q_{1,2,3,4}$ is diagonal. This implies

$$
\begin{aligned}
Q_{e} & =Q_{v}^{-1} Q_{a} Q_{v}, & Q_{f} & =Q_{b}, \\
Q_{g} & =Q_{1}^{-1} Q_{c} Q_{1}, & G_{h} & =\left(Q_{1} Q_{2} Q_{v}\right)^{-1} Q_{d}\left(Q_{1} Q_{2} Q_{v}\right) .
\end{aligned}
$$


In this basis, they should all take the form (A.7), with the associated parameters $\mu_{i}$. Therefore, we define the functions $F_{i j}$ as follows:

$$
\begin{aligned}
& F_{i 1}=\operatorname{Re}\left(Q_{i 1}{ }^{1}-Q_{i 2}^{2}\right), \quad F_{i 2}=\operatorname{Im}\left(Q_{i 1}^{1}+Q_{i 2}^{2}\right), \\
& F_{i 3}=\operatorname{Re}\left(\mu_{i} Q_{i 2}{ }^{1}+Q_{i}^{2}\right), \quad F_{i 4}=\operatorname{Im}\left(\mu_{i} Q_{i 2}^{1}-Q_{i}^{2}\right) .
\end{aligned}
$$

If we choose the $Q_{i}$ here to be $Q_{e, f, g, h}$, these functions should all be zero.

When handling the most general case, the resulting expressions tend to become lengthy. It is more illuminating to take an arbitrary example. We took:

$$
t_{1}=\frac{1}{3}, \quad t_{2}=\frac{1}{2}, \quad v=\frac{1}{7}, \quad w=\frac{1}{5} .
$$

With this, the condition $F_{41}=0$ yields

$$
\begin{aligned}
y_{11}= & \frac{1}{148988448}\left(-3 x_{12}\left(-39692619+46224949 \mu_{2}\right)\right. \\
& \left.+28\left(-3849888+y_{12}\left(33449+2251121 \mu_{2}\right)\right)\right) .
\end{aligned}
$$

Then, $F_{42}=0$ leads to

$$
y_{12}=-\frac{15\left(179661440+x_{12}\left(-75340697+354247143 \mu_{2}\right)\right)}{4\left(-902444771+502645021 \mu_{2}\right)} .
$$

Next came the surprise: requiring $F_{43}=F_{44}=0$ does not fix the value of $x_{12}$, but in stead the value of $\mu_{4}$ :

$$
\mu_{4}=\frac{224726999641+40360716889 \mu_{2}}{133691328551-379277148121 \mu_{2}} .
$$

Indeed, with this value for $\mu_{4}$, the value of $x_{12}$ is kept free. As a check, we find that, with (A.11) and (A.12), all internal holonomies obey the string equation $\operatorname{Im} \operatorname{Tr}\left(Q_{a, b, c, d}\right)=0$. Instead of $x_{12}$, we could choose now $\mu_{1}$ as a new parameter. Therefore, we check the functions $F_{1 i}$. Of these, $F_{11}$ and $F_{12}$ are already zero. Both equations $F_{13}=0$ and $F_{14}=0$ lead to the same expression

$$
x_{12}=\frac{-931364904960\left(-1+\mu_{1}\right)}{1458158337341-2431046249155 \mu_{2}+17 \mu_{1}\left(39825058285+73046360557 \mu_{2}\right)} .
$$

This leaves the functions $F_{3 i}$ to be checked. Again, $F_{31}$ and $F_{32}$ are already obeyed. The remaining two both give the same result:

$$
\mu_{3}=\frac{379277148121+40360716889 \mu_{1}}{133691328551-224726999641 \mu_{1}} .
$$

Since, in this case, both (A.13) and (A.15) have a minus sign in their denominators, it is easy to find positive values for $\mu_{1}$ and $\mu_{2}$ such that both $\mu_{3}$ and $\mu_{4}$ are positive 
as well:

$$
\mu_{1}=\frac{1}{2}, \quad \mu_{2}=\frac{1}{4}, \quad \mu_{3}=\frac{266305004377}{14218552487}, \quad \mu_{4}=\frac{939268715453}{155488166083} .
$$

The newly opened strings indeed also obey (A.6):

$$
\begin{array}{ll}
\operatorname{Tr}\left(Q_{a}\right)=1.68394, & \operatorname{Tr}\left(Q_{b}\right)=1.58638, \\
\operatorname{Tr}\left(Q_{c}\right)=1.88194, & \operatorname{Tr}\left(Q_{d}\right)=1.37714,
\end{array}
$$

where the values were rounded for clarity.

This good behavior, however, is due to the fact that the scattering is at high angles and non-relativistic. If we do the same calculation for slightly different values:

$$
t_{1}=\frac{1}{3}, \quad t_{2}=\frac{1}{2}, \quad v=\frac{4}{7}, \quad w=\frac{4}{5},
$$

rwe get as our two equations:

$$
\begin{aligned}
& \mu_{3}=-\frac{23654969982136936+20734925253590287 \mu_{1}}{19234905848692273+16644373216740712 \mu_{1}}, \\
& \mu_{4}=-\frac{16644373216740712+20734925253590287 \mu_{2}}{19234905848692273+23654969982136936 \mu_{2}},
\end{aligned}
$$

here, we see two equations that both are incompatible with positive values for $\mu_{1}$, $\mu_{3}, \mu_{2}$ and $\mu_{4}$. As stated earlier, the general expressions for all values of $t_{1}, t_{2}, v$ and $w$ in the allowed regions are too lengthy to be revealing.

We here observe that the coefficients $A, B, C$ and $D$ for the two diagonals are clearly related. This is due to the symmetry of the problem:

$$
\text { If } \mu_{1}=\frac{1}{\mu_{4}} \text { then } \mu_{3}=\frac{1}{\mu_{2}} \text {. }
$$

This symmetry is due to the fact that our initial configuration was one with free strings approaching one another. In this case, the existence of positive solutions for $\mu_{1}$ and $\mu_{3}$ automatically guarantees the existence of positive solutions for $\mu_{2}$ and $\mu_{4}$, and vice versa.

\section{References}

1. Staruszkiewicz, A.: Gravity theory in three dimensional space. Acta Phys. Pol. 24, 734 (1963)

2. Aichelburg, P.C., Sexl, R.U.: On the gravitational field of a massless particle. Gen. Relativ. Gravit. 2, 303 (1971)

3. Deser, S., Jackiw, R., 't Hooft, G.: Three dimensional Einstein gravity: dynamics of flat space. Ann. Phys. 152, 220 (1984)

4. 't Hooft, G.: Cosmology in $2+1$ dimensions. Nucl. Phys. B 30, 200 (1993)

5. 't Hooft, G.: The evolution of gravitating point particles in $2+1$ dimensions. Class. Quantum Gravity 10, 1023 (1993) 
6. Gott, J.R.: Phys. Rev. Lett. 66, 1126 (1991)

7. Ori, A.: Phys. Rev. D 44, R2214 (1991)

8. Gödel, K.: An example of a new type of cosmological solution of Einstein's field equations of gravitation. Rev. Mod. Phys. 21, 447 (1949)

9. Deser, S., Jackiw, R., 't Hooft, G.: Physical cosmic strings do not generate closed timelike curves. Phys. Rev. Lett. 68, 267 (1992)

10. Kadar, Z.: Polygon model from first order gravity. Class. Quantum Gravity 22, 809 (2005). e-Print: gr-qc/0410012

11. Witten, E.: $(2+1)$-dimensional gravity as an exactly soluble system. Nucl. Phys. B 311, 46 (1988)

12. Carlip, S.: Six ways to quantize $(2+1)$-dimensional gravity. Can. Gen. Rel. 0215-234, (1993) (QC6:C25:1993), gr-qc/9305020

13. 't Hooft, G.: Quantization of point particles in $(2+1)$ dimensional gravity and spacetime discreteness. Class. Quantum Gravity 13, 1023 (1996), gr-qc/9607022

14. Barret, J.W., et al.: A parallelizable implicit evolution scheme for Regge calculus. Int. J. Theor. Phys. 36, 815 (1997), gr-qc/9411008, and references therein

15. Brandenberger, R., Firouzjahi, H., Karouby, J.: Lensing and CMB anisotropies by cosmic strings at a junction. arXiv:0710.1636 (gr-qc)

16. 't Hooft, G.: A mathematical theory for deterministic quantum mechanics. J. Phys. Conf. Ser. 67, 012015 (2007), quant-ph/0604008 